contradicting prognoses or treatments on the basis of biopsies from different areas. Charles Swanton at Cancer Research UK's London Research Institute and his colleagues analysed this diversity by reconstructing the evolution of four kidney cancers, sampling several parts of each primary tumour, as well as secondary ones from organs to which the cancer had spread.

In one patient, only onethird of the total mutations were found in all the samples, and one-quarter were unique to just one. This has implications for biomarker analysis and cancer-genome studies, which rely on single biopsies. It might also explain why many cancer treatments eventually stop working: they affect only part of a tumour, allowing the remainder to bounce back. N. Engl. J. Med. 366, 883-892 (2012)

\section{PLANETARY SCIENCE}

\section{Venusian hot flow anomalies}

A giant aneurysm of plasma distorted Venus's atmosphere on 22 March 2008, a spaceweather event known as a hot flow anomaly (HFA). Such events occur when electric fields associated with the Sun's solar wind create a fastgrowing bulge that is filled with hot ions from the bow shock, a boundary between the solar wind and the planet.

Venus joins Earth, Saturn and Mars as planets where HFAs have been observed, say Glyn Collinson at the Goddard Space Flight Center in Greenbelt, Maryland, and his colleagues, who used data from the European Space Agency's Venus Express satellite to identify the 2008 event.

On Earth, HFAs can cause auroras. The authors suggest that HFAs would be even more disruptive to Venus's atmosphere because the planet lacks a magnetic field, meaning that the bow shock, and so the HFA, would be much closer in. 10.1029/2011JA017277 (2012)
IMMUNOLOGY

\section{Controlling natural killers}

Immune cells long considered lowly foot soldiers on the front line against bacteria and fungi turn out to have a commanding role. Researchers report that neutrophils, the most common white blood cells in humans, control the activity of another antimicrobial white blood cell — the natural killer cell.

Sophie Ugolini and Eric Vivier of Aix-Marseilles University in France and their colleagues isolated a mutant mouse whose natural killer cells responded poorly to stimulation. The mutant, they found, lacked mature neutrophils. Without neutrophils to guide them, mouse natural killer cells failed to mature or function properly. The same was true of natural killer cells in human patients with low neutrophil counts, a condition known as neutropenia.

J. Exp. Med. http://dx.doi. org/10.1084/jem.20111908 (2012)

\section{PALAEOECOLOGY \\ What killed the big beasts?}

During the past 100,000 years, many of Earth's largest animals - including ground sloths, mammoths, woolly rhinos and sabre-toothed cats - became extinct. Scientists have debated why for decades, with climate change and hunting by humans the chief suspects. Graham Prescott, David Williams and their group at the University of Cambridge, UK, have created a model of unprecedented geographical breadth - and concluded that it took both factors to seal the beasts' fate.

The researchers modelled extinctions in North America, South America, Palaeoarctic Eurasia, Australia and New Zealand, running simulations with climate data from ice cores and thousands of plausible combinations of humanarrival and species-extinction

COMMUNITY CHOICE

The most viewed papers in science

\title{
James Crow on sabotaging sperm
}

\section{$\checkmark$ Highly Read \\ on plosgenetics. org 5 Feb-6 Mar}

A recent paper identifies a cause for sperm with puzzlingly high rates of mutation that cause a particular thyroid cancer syndrome. But the Perspective that accompanied it was viewed more often than the work itself, perhaps because its author, James Crow, a distinguished geneticist at the University of Wisconsin, Madison, died in January, aged 95.

Those who read Crow's last paper found the cause of the high mutation rate summarized in his clear and pleasant prose. Rather than dividing asymmetrically - to produce one sperm and one proto-sperm cell like the parent - some cells 'cheat' and produce two proto-sperm cells instead. Each of these can divide asymmetrically, doubling the cheat's number of descendants and creating clusters of mutations linked to the effect.

PLoS Genet. 8, e1002535 (2012)

For an obituary of James Crow, see go.nature.com/ty6xi5

times, reflecting the large uncertainty in both these estimates. The recipe that best predicted the pattern of extinctions included both climatic and human ingredients.

Proc. Natl Acad. Sci. USA http://dx.doi.org/10.1073/ pnas.1113875109 (2012)

\section{MATERIALS}

\section{A new way to 3D shapes}

Materials that can change their shape on demand have many applications, and, thanks to a team in the United States, an extra means of production. Christian Santangelo, Ryan Hayward and their colleagues at the University of Massachusetts at Amherst report that they have devised a way to produce polymer sheets that can transform into predetermined three-dimensional shapes and back again.

The researchers created gel sheets from polymers of $\mathrm{N}$-isopropylacrylamide and benzophenone, a molecule that creates crosslinking between polymers when exposed to ultraviolet (UV) light. These sheets swell in

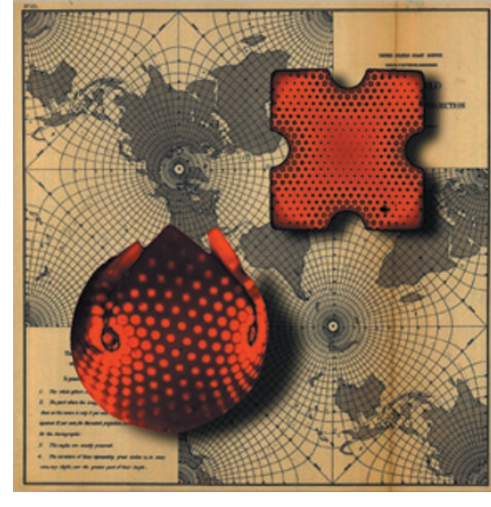

water, but shrink when heated.

The team patterned the gel using a mask and an initial low dose of UV to define the polymer's overall shape, then used a higher dose through a different mask to create highly crosslinked regions in which swelling is reduced. By allowing more swelling in some areas than others, the authors created objects that can switch between sheets and spheres when heated and cooled (pictured), as well as others that can reversibly form caps, cones and morecomplex surfaces.

Science 335, 1201-1205 (2012)

\section{$\rightarrow$ NATURE.COM}

For the latest research published by Naturevisit:

www.nature.com/latestresearch 